\title{
DISEÑO Y VALIDACIÓN DEL INVENTARIO DE DEPENDENCIA EMOCIONAL - IDE
}

DESIGN AND VALIDATION OF EMOTIONAL DEPENDENCY INVENTORY - IDE

\author{
JeSÚs AIQUIPA T. ${ }^{1}$ \\ Universidad Nacional Mayor de San Marcos, Lima, Perú \\ (RECIBIDO 8/1/2012; ACEPTADO 25/6/2012)
}

\begin{abstract}
RESUMEN
El propósito de esta investigación fue construir y validar un instrumento que reúna las propiedades psicométricas adecuadas para medir la dependencia emocional. La muestra total estuvo conformada por 757 participantes de Lima-Perú (398 mujeres y 359 varones), con edades entre los 18 y 55 años. Del total de reactivos iniciales, fueron eliminados más del $75 \%$ de ellos a través de procedimientos sistemáticos, quedando la prueba final conformada por 49 items. Los resultados muestran que el Inventario de Dependencia Emocional (IDE), tiene indicadores de confiabilidad y evidencias de validez de constructo adecuados.
\end{abstract}

Palabras clave: Inventario, dependencia emocional, confiabilidad, validez de constructo.

\begin{abstract}
The purpose of this research was to construct and validate an instrument to meet adequate psychometric properties to measure the emotional dependency. The total sample consisted of 757 participants from Lima-Peru (398 women and 359 men) aged between 18 and 55 . Of total initial items were eliminated over $75 \%$ of them through systematic procedures, leaving the final test consisted of 49 items. The results show that Emotional Dependency Inventory - IDE has confiabiliadad indicators and evidence of adequate construct validity.
\end{abstract}

Keywords: Inventory, emotional dependency, reliability, construct validity. 


\section{INTRODUCCIÓN}

Las relaciones afectivas saludables que se establece con una pareja son una fuente inagotable de bienestar para los miembros que la conforman. Es una expresión más de salud mental que un individuo goza en una época determinada de su vida.

Es normal que en una relación de pareja, en particular durante la fase del enamoramiento, se sienta una necesidad afectiva intensa con el otro. Pero este deseo, a medida que la relación de pareja se estabiliza, tiende a disminuir para conformar una relación madura, donde el amor y sus componentes están en un equilibrio que propicia el desarrollo personal de los miembros de la pareja (Avendaño y Sáchez, 2002; Riso, 2003). En cambio, cuando el deseo de fusión permanece inalterado con el pasar del tiempo y es más, aumenta, cuando hay una necesidad afectiva intensa de estar con la pareja recurriendo a cualquier acto -en muchos casos perjudicial para uno de los miembros-, la dependencia emocional se convierte en la principal, y tal vez única razón de ser la relación.

Este problema es poco conocido, pero es frecuente en la actividad clínica y en muchos casos son la base de algunos trastornos afectivos (Riso, 2003; Castelló, 2005; Sirvent y Moral, 2007). La mitad de la consulta psicológica se debe a problemas ocasionados o relacionados con dependencia patológica interpersonal, principalmente con la pareja. En muchos casos, pese a lo nocivo de la relación, las personas son incapaces de ponerle fin a la misma (Riso, 2003). Sirvent y Moral (2007) confirman que un alto porcentaje de la población española se declara dependiente emocional $(49.3 \%)$ y que en su versión más severa puede llegar a afectar nada menos que a un $8.6 \%$ (Sirvent, Moral, Blanco y Palacios, 2004; citado en Sirvent, 2006).

En relación al género, Lazo (1998) manifiesta que el mayor porcentaje de personas con este problema son mujeres, de diferentes clases sociales, atractivas, inteligentes, con ocupaciones diversas, trabajadoras dependientes e incluso amas de casa. Algunas de ellas inclusive opuestas a conductas machistas, por lo menos verbalizando tal expresión. Sin embargo, todas ellas dependientes o necesitadas de "amor" de parte de sus parejas. Castelló (2005) concuerda con esta observación y manifiesta que en lo que se refiere al género, la confluencia entre factores biológicos y socioculturales otorga a la mujer una mayor probabilidad de que padezca dependencia emocional.

De forma general, el constructo objeto del siguiente estudio ha sido solapado -y hasta confundido- por otros conceptos, que cuentan con mayor investigación científica (tal como es la dependencia instrumental, cuyas características configuran el trastorno de personalidad por dependencia [Castelló, 2005]), son genéricos (por ejemplo, dependencias interpersonales) o definen conceptos distintos (verbigracia, codependencia). En este mismo sentido, puede entenderse entonces que la dependencia emocional casi no es tomada en cuenta en los estudios psicométricos, y si lo es, se consideran solo algunos aspectos del mismo.

Tales son así algunas pruebas que cuentan con mayor rigor científico como la Escala de Dependencia Interpersonal (Interpersonal Dependency Inventory - IDI), creada 
por Hirschfeld et al en 1977; la Escala de Dependencia Específica en la Pareja (Spouse Specific Dependency Scale - SSDS), elaborada por Rathus y O'Leary, en 1997; y el Cuestionario de Dependencia Emocional - CDE de Lemos y Londoño (2006), siendo este último una prueba que mide esta realidad pero ha sido creada desde un marco teórico distinto al planteado en este estudio.

En el Perú, no se ha abordado este constructo para elaborar una prueba o escala que mida la dependencia emocional. Se han realizado algunos estudios que relacionan esta variable con la violencia doméstica o de pareja, utilizando entrevistas clínicas (Lazo, 1998; Castro, 2001) o pruebas adaptadas que miden dependencia psicológica por consumo de sustancias psicoactivas (Loayza, 2002).

\section{Modelo de vinculación afectiva}

Castelló (2005) plantea su modelo teórico de la vinculación afectiva para explicar la naturaleza de la dependencia emocional. Aquella es definida como la unión de un sujeto con otras personas, y la necesidad de crear y mantener lazos permanentes con ellas. Además, esta vinculación afectiva con los demás puede darse en diferentes grados, dado que conforma un hipotético continuo.

Así, las personas con relaciones interpersonales sanas, se ubicarían dentro del área de la vinculación pero en un grado medio. Se concluye entonces que la diferencia entre las relaciones de parejas normales y las que lleva a cabo un dependiente emocional son cuantitativas, y por ello se podrían ubicar en un hipotético continuo, manifestándose en grados o niveles de vinculación afectiva.

\section{Definición de dependencia emocional}

La dependencia emocional es un tipo de dependencias relacionales genuinas (Sirvent, 2006) y es definida como la dimensión disfuncional de un rasgo de personalidad, que consiste en la necesidad extrema de orden afectiva que una persona siente hacia otra, su pareja, a lo largo de sus diferentes relaciones de pareja (Castello, 2000, 2005). Esta definición tiene una perspectiva distinta a la que plantean otros autores, definiendo la dependencia emocional como un tipo de adicción (Schaeffer, 1998; Lazo, 1998; Riso, 2003).

En función a lo revisado y ante la carencia de pruebas psicométricas que puedan medir el constructo dependencia emocional de forma explícita y específica, basado en un modelo teórico sistematizado y consistente con la teoría de los rasgos (Cloninger, 2003), tal como lo es el modelo que propone Castelló (2005), el propósito de la siguiente investigación radica en la construcción de un inventario que cumpla con las condiciones psicométricas adecuadas para medir la dependencia emocional.

\section{METODOLOGÍA}

\section{Diseño de investigación}

El siguiente estudio se desarrolla bajo un enfoque cuantitativo, psicométrico y de tipo correlacional. El diseño de investigación es no experimental, del tipo transversal y comparativo. 


\section{Población y muestra}

La población está constituida por varones y mujeres, con edades comprendidas entre los 18 y 55 años, de diferente nivel socioeconómico, que radiquen en la ciudad metropolitana de Lima, con un nivel de comprensión lectora adecuado (equivalente a seis años de escolaridad básica), que no sufran trastornos mentales o causados por enfermedad (psicosis, demencia, intoxicación medicamentosa o sedados, etc.) que afecten su habilidad para cumplimentar inventarios de autoinforme, y que hayan tenido por lo menos una relación de pareja (heterosexual u homosexual) a lo largo de su vida. El muestreo es no probabilístico, de tipo intencional. La muestra total estuvo conformada por 757 sujetos, entre estudiantes universitarios y empleados/ as (dependientes e independientes laboralmente). Se trabajó con 4 muestras en las diferentes etapas del estudio. La primera muestra estuvo representada por 126 sujetos, la cual sirvió para el estudio "Pre-Piloto". La segunda muestra, designada para el Estudio "Piloto", estuvo conformada por 200 personas. La tercera muestra conformó por 31 mujeres que siguen un programa de tratamiento psicológico residencial en un Centro de Atención Clínica, diagnosticadas como dependientes emocionales. Finalmente, la cuarta muestra estuvo conformada por 400 personas de ambos sexos y sirvió para el análisis de confiabilidad y validez final, así como para establecer los baremos.

\section{Instrumento}

Para este estudio se empleó el Inventario de Dependencia Emocional-IDE, el mismo que fue sometido a pruebas rigurosas para su versión final, conformada por 49 ítems agrupados en siete factores.

\section{Procedimiento}

Se elaboró un inventario que comprendió 209 reactivos, tomando como los modelos propuestos del total de bibliografías, así como la información directa obtenida del trabajo con un grupo de pacientes identificadas como dependientes emocionales. El tipo de reactivo utilizado fue de selección de respuesta múltiple, con el formato de una escala Likert de 5 puntos.

Posteriormente, se entregó la versión de la prueba inicial a cinco jueces (tres psicólogos clínicos, un psiquiatra y un psicólogo con conocimiento en la elaboración de pruebas psicométricas). Luego de las observaciones, se redujo la prueba a una versión de 204 reactivos. Paralelamente, se enviaron las cartas de presentación y solicitudes a aglunas las instituciones académicas participantes (una universidad pública y dos instituciones educativas). Asimismo, se envió otra solicitud al Centro de tención Clínica para la aplicación de la prueba a las pacientes residentes. Se llevó a cabo los estudios Prepiloto y Piloto para el análisis de los items. Luego,

se aplicó la prueba final a las 31 mujeres que siguen un programa de tratamiento y a otro grupo no clínico (conformado por 28 sujetos de la muestra final). Posteriormente, se aplicó esta misma prueba a una cuarta muestra conformada para obtener los niveles de confiabilidad y validez definitivos, así como los baremos. Finalmente, se elaboró el manual del IDE. 


\section{Análisis estadístico}

Se utilizó el programa de cálculo Excel 10.0 y el Programa SPSS, versión 14.

Se utilizó la prueba de Kolmogorov-Smirnov para comprobar si la variable de interés (dependencia emocional, variable cuantitativa, susceptible de ser medida en una escala de intervalo) sigue una distribución normal en las distintas muestras, y de este modo, determinar el tipo de prueba estadística a emplear: paramétrica o no paramétrica. Para todos los análisis que se realizaron, se consideró un nivel de significación estadística del 5\% (0.05).

El coeficiente de confiabilidad se obtuvo por medio de la consistencia interna, a través del coeficiente " $r$ " de Pearson corregida mediante la ecuación Spearman -Brown y el coeficiente de fiabilidad alfa de Cronbach. Para el análisis de la validez de contenido por criterio de jueces se empleó el Índice de Acuerdo (IA). El análisis de la validez de grupos contrastados o de criterio se realizó a través de la comparación de medias, por medio del estadístico "t" de student para grupos independientes. Para el Análisis Factorial (Exploratorio), se empleó el método de componentes principales con rotación ortogonal varimax, previa verificación de bondad de ajuste a este análisis (índice de determinación, índice de Kaiser Meyer - Olkin (KMO) y prueba de esfericidad de Bartlett).

\section{RESULTADOS}

\section{Descripción de la muestra}

Como se observa en la Tabla 1, la muestra total estuvo constituida por 757 participantes, 398 mujeres (53\%) y 359 varones (47\%). La media de edad es 22.8 años, con una desviación estándar de 7.1 años. Es el grupo jóvenes, edades comprendidas emtre los 18 y 24 años de edad, donde se registra la mayor frecuencia: 623 sujetos que representan el $82 \%$. La mayoría de los participantes tienen un nivel de instrucción superior universitario $(77.4 \%)$ y se dedican a labores académicas $(79.3 \%)$.

Tabla 1: Estadísticas descriptivas de las variables demográficas de la Muestra Total.

\begin{tabular}{llccc}
\hline \multicolumn{1}{c}{ Variables } & \multicolumn{1}{c}{ Categorías } & N & $\%$ \\
\hline \multirow{2}{*}{ Sexo } & Varón & 398 & 53 \\
& Mujer & 359 & 47 \\
& Jóvenes: 18-24 años & 623 & 82 \\
\multirow{2}{*}{ Edad (_(X=22.8;DE=7.18) } & Adultos Jóvenes: 25-34 años & 76 & 10 \\
& Adultos Medios: 35-44 años & 30 & 4 \\
& Adultos Mayores: 45-55 años & 28 & 4
\end{tabular}




\begin{tabular}{llcc} 
& Primaria & 0 & 0 \\
\multirow{3}{*}{ Nivel de Instrucción Educativa } & Secundaria & 31 & 4.1 \\
& Superior Técnica & 140 & 18.5 \\
& Superior Universitaria & 586 & 77.4 \\
& Estudiante & 600 & 79.3 \\
\multirow{3}{*}{ Ocupación } & Empleado Dependiente & 115 & 15.2 \\
& Empleado Independiente & 42 & 5.5 \\
Relación de Pareja Actual & Sí & 343 & 45.3 \\
& No & 414 & 54.7 \\
\hline & Total & 757 & 100.0 \\
\hline
\end{tabular}

\section{Análisis de Reactivos}

Criterio de Jueces e Índice de Acuerdo como Análisis de ítems. Se consideró apropiado los criterios de 5 jueces, con conocimiento en los temas estudiados. Se usó como indicador numérico el Índice de Acuerdo (IA): $\mathrm{IA}=\mathrm{A} /(\mathrm{A}+\mathrm{D})$; donde A es Juicios que coinciden con la definición propuesta por el autor (Totalidad de Acuerdo) y D es Desacuerdos emitidos. Mediante este análisis, fueron eliminados cinco reactivos por tener un IA menor a 0.80 .

Índice de Homogeneidad o Correlación Ítem-Test. Se obtuvo el coeficiente de correlación de Pearson Corregida, que sirvió como índice de homogeneidad (correlación ítem-total). Los reactivos que obtuvieron un coeficiente de correlación por debajo de 0.50 fueron eliminados. La Tabla 2 muestra estos coeficientes de los reactivos que luego conformarían la prueba final. Es preciso señalar que figuran con sus códigos iniciales.

Tabla 2: Coeficientes de correlación ítem-total de los reactivos de la Prueba Final

\begin{tabular}{cccc}
\hline Items & $\begin{array}{c}\text { Correlación Ítem - Total } \\
\text { Corregido }\end{array}$ & Items & $\begin{array}{c}\text { Correlación Ítem - Total } \\
\text { Corregido }\end{array}$ \\
\hline 45 & .539 & 118 & .516 \\
47 & .537 & 131 & .659 \\
52 & .529 & 135 & .629 \\
53 & .687 & 137 & .688 \\
61 & .532 & 150 & .590 \\
62 & .592 & 151 & .527 \\
67 & .581 & 155 & .676 \\
69 & .597 & 156 & .661 \\
75 & .612 & 158 & .561 \\
76 & .605 & 159 & .607 \\
78 & .630 & 161 & .590 \\
81 & .634 & 163 & .666
\end{tabular}




\begin{tabular}{llll}
86 & .552 & 168 & 523 \\
89 & .564 & 170 & .530 \\
90 & .522 & 175 & .601 \\
92 & .523 & 176 & .636 \\
97 & .594 & 177 & .522 \\
102 & .649 & 182 & .531 \\
107 & .581 & 184 & .545 \\
108 & .600 & 185 & .573 \\
109 & .629 & 186 & .629 \\
110 & .588 & 188 & .554 \\
111 & .669 & 191 & .654 \\
113 & .655 & 204 & .578 \\
117 & .696 & & \\
\hline
\end{tabular}

Análisis factorial como técnica de análisis de ítems. Antes de hacer el análisis factorial, se comprobó los índices de ajuste a este tipo de análisis. El índice de determinación cercano a cero pero no cero, en este caso, 9.36E-022; índice de Kaiser - Meyer - Olkin (KMO) igual a 0.92 y la prueba de esfericidad de Bartlett, significativo estadísticamente al 0.05. Luego, se preseleccionaron 14 factores (autovalor mayor a 1) que explican un total de varianza igual a 68.259\%. Se creyó conveniente eliminar siete factores por tener tres variables o menos, con saturaciones regulares: Factores 8, 9, 10 y 11, todos ellos con tres ítems cada uno; los Factores 12 y 14, con un ítem cada uno y el Factor 14, con dos reactivos. Por tanto, se eliminaron en total 16 reactivos.

\section{Confiabilidad}

La confiabilidad se obtuvo a través del método de consistencia interna, usando los coeficientes Alfa de Crombach y " $r$ " de Pearson corregida con la fórmula Spearman-Brown. En la Tabla 2 aparecen los estadísticos descriptivos principales de los dos pretest y del test final del IDE, así como los valores de los coeficientes alfa de Cronbach. Asimismo, un segundo método que se utilizó para medir la confiabilidad del IDE, por medio de la consistencia interna fue el Método de la división por mitades. El coeficiente de correlación " $r$ " de Pearson, corregido mediante la fórmula Spearman-Brown, fue de 0.91.

Tabla 3: Coeficientes Alfa de Crobanch de los Pretest y la Versión Final del IDE

\begin{tabular}{ccccc}
\hline Pruebas & N $^{\circ}$ Ítems & $-\mathrm{X}$ & $\mathrm{DE}$ & Alfa \\
\hline $1^{\circ}$ Pretest & 204 & 415.93 & 74.801 & 0.968 \\
$2^{\circ}$ Pretest & 86 & 190.78 & 51.054 & 0.975 \\
Test Final & 49 & 103.61 & 31.962 & 0.965 \\
\hline
\end{tabular}




\section{Validez}

Evidencias de validez de contenido. Los cinco expertos mostraron acuerdo en la relevancia de las dimensiones establecidas. De esta manera, más del $95 \%$ de los reactivos fueron considerados apropiados para medir el constructo dependencia emocional por el $100 \%$ de los jueces. Este procedimiento también sirvió para el análisis de reactivos.

Evidencias de validez factorial. Se evaluó la adecuación de las matrices de correlaciones utilizando el índice de adecuación de la muestra de Kaiser- Meyer-Olkin, con un valor satisfactorio de 0.96. El Test de Esfericidad de Barlett fue significativo ( $\mathrm{X}=10969.743 ; \alpha=.000)$, indicativo de la presencia de correlaciones significativas y la pertinencia de llevar a cabo el análisis factorial. Luego, Se extrajeron siete factores que tienen autovalores mayores que 1 y que explican el $58.25 \%$ de la varianza de los datos originales. La Tabla 3 presenta los estadísticos resúmenes básicos de cada uno de los factores.

Tabla 4: Estadísticos principales y coeficientes Alfa de los Factores del IDE.

\begin{tabular}{|c|c|c|c|c|c|}
\hline Factores & _X & $\mathrm{DE}$ & Elementos & $\mathrm{N}^{0}$ Ítems & Alfa \\
\hline Factor 1:Miedo a la ruptura & 15.6 & 6.18 & $\begin{array}{c}5,9,14,15,17,22 \\
26,27,28 .\end{array}$ & 9 & 0.88 \\
\hline $\begin{array}{l}\text { Factor 2: Miedo e Intolerancia a } \\
\text { la soledad }\end{array}$ & 22.5 & 8.06 & $\begin{array}{c}4,6,13,18,19,21 \\
24,25,29,31,46\end{array}$ & 11 & 0.89 \\
\hline Factor 3: Prioridad de la pareja & 16.2 & 6.20 & $\begin{array}{c}30,32,33,35,37 \\
40,43,45\end{array}$ & 8 & 0.86 \\
\hline $\begin{array}{l}\text { Factor } 4 \text { : Necesidad de acceso a } \\
\text { la pareja }\end{array}$ & 14.3 & 5.11 & $\begin{array}{c}10,11,12,23,34 \\
48 .\end{array}$ & 6 & 0.87 \\
\hline $\begin{array}{l}\text { Factor 5: Deseos de exclusivi- } \\
\text { dad }\end{array}$ & 10.6 & 4.19 & $16,36,41,42,49$ & 5 & 0.79 \\
\hline $\begin{array}{l}\text { Factor 6: Subordinación y Sumi- } \\
\text { sión }\end{array}$ & 12.3 & 4.07 & $1,2,3,7,8$ & 5 & 0.76 \\
\hline $\begin{array}{l}\text { Factor 7:Deseos de control y } \\
\text { dominio }\end{array}$ & 11.8 & 4.08 & $20,38,39,44,47$ & 5 & 0.77 \\
\hline
\end{tabular}

Evidencias de validez por diferencias de grupos contrastados. Para encontrar este tipo de evidencia se aplicó la prueba final a un grupo de 31 mujeres que fueron diagnosticadas como dependientes emocionales y que recibían tratamiento psicológico de tipo residencial, siendo dicho problema uno de los más relevantes. También se aplicó a otro grupo no clínico (tomada de la muestra final). Posterior a la realización de la base de datos para este procedimiento, se compararon las medias de ambos grupos utilizando la prueba "t" de Student. Estos datos aparecen en la Tabla 4. Asimismo, la Tabla 5 muestra la diferencia de medias según el sexo tanto a nivel de Factores como de la Prueba Total. 
Tabla 5: Diferencias de medias entre grupo Clínico y Normal.

\begin{tabular}{lcccccc}
\hline \multicolumn{1}{c}{ Muestra } & $\mathrm{N}$ & $-\mathrm{X}$ & $\mathrm{DE}$ & $\mathrm{Dif}$. & $\mathrm{T}$ & Sig. \\
\hline Clínica & 31 & 170.4 & 32.7 & & & \\
No Clínica & 28 & 64.8 & 7.9 & 105.6 & 17.43 & 0.000 \\
\hline
\end{tabular}

Tabla 6: Diferencia de medias entre varones y mujeres por Factores y Escala Total.

\begin{tabular}{|c|c|c|c|c|c|c|c|}
\hline \multirow{2}{*}{ Factores } & \multicolumn{2}{|c|}{ Mujeres (N: 208 ) } & \multicolumn{2}{|c|}{ Varones (N: 192) } & \multirow{2}{*}{ Dif. } & \multirow{2}{*}{$\mathrm{T}$} & \multirow{2}{*}{ Sig. } \\
\hline & - X & $\mathrm{DE}$ & ${ }_{-} \mathrm{X}$ & $\mathrm{DE}$ & & & \\
\hline 1 & 16.7 & 6.1 & 14.6 & 6.1 & 2.1 & 3.37 & 0.001 \\
\hline 2 & 23.4 & 8.1 & 21.6 & 8.0 & 1.8 & 2.23 & 0.026 \\
\hline 3 & 17.4 & 6.2 & 15.0 & 5.9 & 2.4 & 3.96 & 0.000 \\
\hline 4 & 14.7 & 4.8 & 13.9 & 5.4 & 0.8 & 1.50 & 0.134 \\
\hline 5 & 11.3 & 4.0 & 10.0 & 4.2 & 1.3 & 3.24 & 0.001 \\
\hline 6 & 13.2 & 3.9 & 11.5 & 4.1 & 1.7 & 4.18 & 0.000 \\
\hline 7 & 12.1 & 3.9 & 11.5 & 4.3 & 0.6 & 1.58 & 0.114 \\
\hline Total & 108.6 & 31.9 & 97.7 & 33.4 & 10.9 & 3.33 & 0.001 \\
\hline
\end{tabular}

\section{Baremos}

La Tabla 6 muestra las medias, desviaciones estándar y normas en percentiles que se obtuvieron con esta última muestra, tanto por factores como a nivel de la Escala Total. También se presentan las categorías diagnósticas.

\section{DISCUSIÓN}

En relación al análisis de ítems, se obtuvieron reactivos con un índice de correlación rit igual o superiores a 0.50 , puesto que el rasgo que se intenta medir (dependencia emocional) está definido de modo riguroso y específico y se garantiza una mejor correlación entre los reactivos para el análisis factorial.

Luego, cuando se procesaron estos elementos mediante el Análisis Factorial, se extrajo 14 factores iniciales que explicaron un total de $68.25 \%$ de la varianza. Dado que 7 de ellos presentaron un máximo de 3 ítems con carga regulares, se decidió eliminar dichos reactivos y por ende los factores que los contenían.

Si bien después de este proceso se retuvieron siete (7) factores que explicaron el $55.981 \%$ de la varianza total, aún no se podía concluir que la prueba final tenga esa cantidad de componentes y reactivos, puesto que aún faltaba determinar la reproducción de los mismos en la muestra final. 


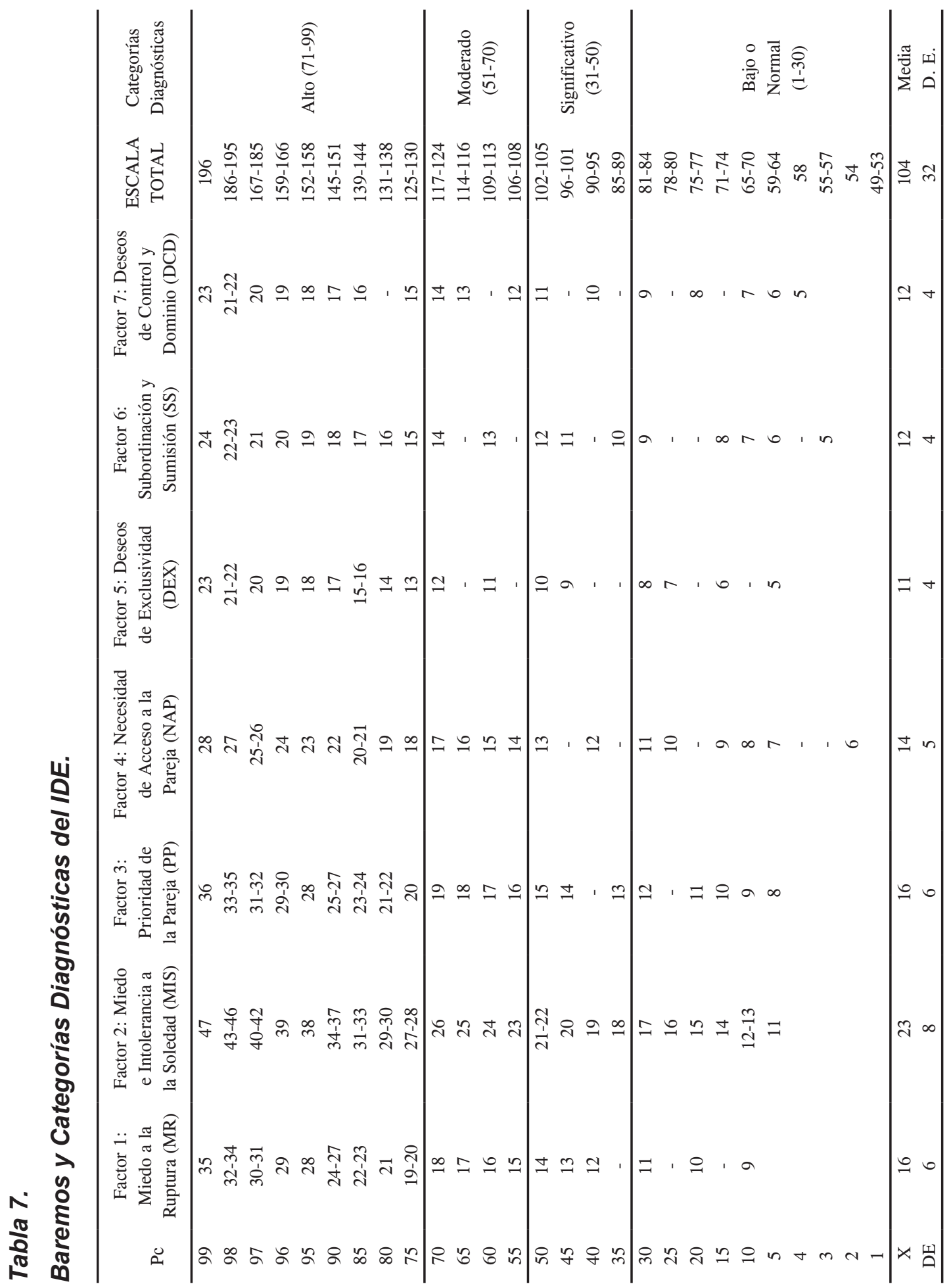


Se obtuvo evidencias de validez de contenido elaborando una escala con 209 reactivos, tomando en cuenta la información disponible de las fuentes ya mencionadas. Se obtuvieron los índices de Acuerdo, con valores de 0.80 y 1.00 para más del $95 \%$ de los reactivos.A su vez, se encontró evidencias de validez factorial al realizarse el Análisis Factorial Exploratorio de la prueba final. Al realizarse este procedimiento, se obtuvieron 7 factores que explicaron el $58.25 \%$ de la varianza total, conteniendo ítems con saturaciones entre 0.40 y 0 . 70. Asimismo, los factores hallados tienen coeficientes de confiabilidad Alfa de Cronbach satisfactorios, que van desde 0.76 hasta 0.89 . Estos mismos fueron nombrados en función al contenido de sus ítems, además de basarse en las características descritas por Castelló $(2000,2005)$ y refieren los siguientes procesos:

1. Miedo a la ruptura - MR (9 ítems): Temor que se experimenta ante la idea de disolución de la relación, adoptándose conductas para mantener la relación. Negación cuando se hace realidad una ruptura, ejerciendo continuos intentos para reanudar la relación.

2. Miedo e Intolerancia a la soledad - MIS (11 ítems): Sentimientos desagradables experimentados ante la ausencia momentánea o definitiva de la pareja. Tendencia a retomar la relación o buscar otra lo más pronto posible para evitar la soledad.

3. Prioridad de la pareja - PP (8 ítems): Tendencia a mantener en primer lugar de importancia a la pareja sobre cualquier otro aspecto o personas.

4. Necesidad de acceso a la pareja - NAP (6 ítems): Deseos de tener presente a la pareja en todo momento, ya sea físicamente o mediante pensamientos.

5. Deseos de exclusividad - DEX (5 ítems): Tendencia a enfocarse en la pareja y aislarse paulatinamente del entorno, acompañada deseos de reciprocidad de esta conducta por la pareja.

6. Subordinación y sumisión - SS (5 ítems): Sobreestimación de las conductas, pensamientos, sentimientos e intereses de la pareja, acompañada sentimientos de inferioridad y desprecio hacia uno mismo.

7. Deseos de control y dominio - DCD (5 ítems): Búsqueda activa de atención y afecto para captar el control de la relación de pareja, a fin de asegurar su permanencia.

Por otro lado, la escala total discrimina entre las medias del grupo clínico $(X=170.4, D E=32.7)$ y no clínico $(X=64.8, D E=7.9), t(17.46)=105.6$, $p<.005$. Ello indicaría, como lo manifiestan Lazo (1998); Castelló (2000, 2005); Riso (2003); Moral (2006) y Sirvent y Moral (2007), que este problema es un motivo de consulta observado muy frecuente en las personas que son asistidas en la práctica clínica. En esta misma línea, las mujeres tienden a puntuar más alto $(X=108.6, D E=31.9)$ que los varones $(X=97.7, D E=33.4)$ en el puntaje total, 
$t(3.33)=10.9, p<.005$, confirmando observaciones realizadas por Lazo (1998), Riso (2003), Castelló (2005) y Sirvent y Moral (2007) También se encontró este patrón en todos los factores a excepción de los factores Necesidad de acceso a la pareja y Deseos de control y dominio.

\section{CONCLUSIONES}

1. El Inventario de Dependencia Emocional (IDE) es una prueba de dominio específico y se empleó métodos combinados para su elaboración (Hogan, 2004).

2. El IDE posee reactivos con índices de correlación igual o superiores a 0.50.

3. El IDE es confiable por medio del método de consistencia interna.

4. El IDE posee pruebas de validez de constructo puesto que se encontró evidencias de validez de contenido, validez factorial y validez de grupos contrastados.

5. Los factores que conforman el IDE, sus etiquetas y contenidos son similares a los propuestos por Castelló (2005). Así también, uno de ellos, Miedo a la soledad, es parecido en nombre y contenido al encontrado por Lemos y Londoño (2006).

6. Los puntajes directos obtenidos en el IDE han sido transformados a puntajes percentilares para la Escala Total y sus siete Factores, identificándose cuatro categorías diagnósticas: bajo o normal, significativo, moderado y alto.

7. Se sugiere la reproducción del estudio con muestras más grandes para afianzar la validez de constructo e investigar la relación que pueda tener la dependencia emocional con otras variables (violencia de pareja, trastornos afectivos, etc.)

\section{NOTA DE RECONOCIMIENTO}

El autor agradece al Consejo Superior de investigaciones de la UNMSM por el financiamiento del estudio. Así también, al Dr. Jaime Ramiro Aliaga Tovar, por su denodada asesoría. A los psicólogos: Karen Gutiérrez S., Natalia Ramírez S., Renato Santibáñez O., Edgar Herrera F., Ana Delgado V., José Chávez Z. y Ricardo Oliveros M., por el apoyo, motivación y sugerencias brindadas en su momento. Asimismo, al equipo terapéutico de la Institución "Vida Mujer" por su colaboración mediante alcances pertinentes.

\section{REFERENCIAS BIBLIOGRÁFICAS}

Avendaño, A. y Sáchez, J. (2002). La dependencia emocional en las parejas, Revista Intercontinental de Psicología y Educación, 4 (1), 91-97.

Castro, A. (2001). La dimensión afectiva en las mujeres afectadas por la violencia familiar. Perú: Ministerio de Salud. 
Castelló, J. (2000). Análisis del concepto "dependencia emocional". I Congreso Virtual de Psiquiatría. Recuperado el 14 de Julio del 2007, de http://www.psiquitria.com/ congreso/mesas/mesa6/conferencias/6_ci_a.htm.

Castelló, J. (2005). Dependencia Emocional: Características y Tratamiento. España: Alianza Editorial.

Cloninger, S. (2003). Teorías de la personalidad. México: Pearson Educación

Hirschfeld, R.M.A.; Klerman, G.L.; Gough, H.G.; Barrett, J.; Korchin, S.J. y Chodoff, P. (1977). A measure of interpersonal dependency. Journal of Personality Assess ment, 41 (6), 610-618.

Hogan, T. (2004). Pruebas psicológicas. Una introducción práctica. México: Manual Moderno.

Lazo, J. (1998). Adicción al amor. Revista de Psicología. Universidad Inca Garcilaso de la Vega, 2 (1-2), 17-29.

Lemos, M. y Londoño, N. (2006). Construcción y validación del Cuestionario de Dependencia Emocional en población colombiana. Acta Colombiana de Psicología. Universidad de San Buenaventura. 9,(2), 127-140.

Loayza, W. (2002). La dependencia psicológica en las mujeres víctimas de violencia en la relación de parejas usuarias de la Defensoría Parroquial Lesttonacc de la ciudad de Chepen - La Libertad. Tesis no publicada, Universidad Nacional Mayor de San Marcos, Lima, Perú.

Rathus, J.H. y O'Leary, K.D. (1997). Spouse-Specific Dependency Scale: Scale Development. Journal of Family Violence, 12 (2), 159-168.

Riso, W. (2003). ¿Amar o depender? Bogotá: Grupo Editorial Norma.

Schaeffer, B. (1998). ¿Es amor o adicción? Barcelona: Apóstrofe.

Sirvent, C (2006). Clasificación y sintomatología diferencial de las dependencias sentimentales y coadicciones. Las dependencias relaciónales: dependencia emocional, codependencia y bidependencia. Memorias del I Encuentro Profesional sobre Dependencias Sentimentales. Fundación Instituto Spiral. Recuperado el 17 de agosto del 2007, de http://www.insitutospiral.com/ cursosyseminarios/encuentros/ encuentros/resumenes/Carlos\%20Sirvent.pdf.

Sirvent, C. y Moral, M. (2007) La dependencia sentimental o afectiva. $8^{\circ}$ congreso virtual de Psiquiatría, "Interpsiquis 2007". Recuperado el 18 de agosot del 2007, de http:// www.apfem.com/articulodelmes/07-07 dependencia\%20sentimental\%20 o\%20afectiva.pdf. 
